\title{
A Mobile Feedback System for Integrated E-health Platforms to improve Self-Care and Compliance of Diabetes Mellitus Patients
}

\author{
A. Fioravanti, G. Fico, M.T. Arredondo, J.-P. Leuteritz
}

\begin{abstract}
Exploiting the full potential of telemedical systems means using platform based solutions: data are recovered from biomedical sensors, hospital information systems, care-givers, as well as patients themselves, and are processed and redistributed in an either centralized or, more probably, decentralized way. The integration of all these different devices, and interfaces, as well as the automated analysis and representation of all the pieces of information are current key challenges in telemedicine.

Mobile phone technology has just begun to offer great opportunities of using this diverse information for guiding, warning, and educating patients, thus increasing their autonomy and adherence to their prescriptions. However, most of these existing mobile solutions are not based on platform systems and therefore represent limited, isolated applications.

This article depicts how telemedical systems, based on integrated health data platforms, can maximize prescription adherence in chronic patients through mobile feedback. The application described here has been developed in an EU-funded R\&D project called METABO, dedicated to patients with type 1 or type 2 Diabetes Mellitus.
\end{abstract}

\section{INTRODUCTION}

Integrated medical information systems are considered to be increasingly important for patients with chronic diseases [1]. Telemedicine for chronic diseases serves the primary goal of improving the patient's health by empowering him/her to follow his prescriptions [2]. Thus, the concept of telemedicine involves shifting the role of the patient from a passive receiver of medical attention to an autonomous manager of his/her own health condition, well informed about the key factors of his/her well-being [3]. As the main method of telemedicine that serves to achieve this goal lies in providing added value through improved information and communication [4], a key factor of this empowerment lies in the feedback given to the patient by the medical Information and Communication Technology (ICT).

Within this article, we will point out the main issues in supporting this empowerment through telemedical applications and describe possible solutions, using as an example the METABO patient feedback system.

Manuscript received April 15,2011. This work is partially funded by the European Commission through the METABO Project, "Controlling Metabolic Diseases Related to Metabolic Disorders", FP7-ICT-2007-1216270 .

A. Fioravanti, G. Fico, and M.T. Arredondo are with the Life Supporting Technologies group, Technical University of Madrid, Escuela Técnica Superior de Ingenieros de Telecomunicación, ES 28040 (phone: +34915495700; author emails: afioravanti@1st.tfo.upm.es, gfico@1st.tfo.upm.es, mta@1st.tfo.upm.es).

J.-P. Leuteritz works at the Institute for Human Factors and Technology Management at the University of Stuttgart, Germany (janpaul.leuteritz@iao.fraunhofer.de)
METABO is an R\&D project, co-funded by the European Commission [5]. Its aim is the development of a system to assist diabetic patients in controlling their metabolic disorders. METABO is not a single application; it is a platform based system, which intends to provide full connectivity in order to be integrated in existing information systems, e.g. in hospitals. One of the main objectives of the METABO platform is to help the patients follow an adequate care pathway by giving medically approved advice and by providing instant and personalized recommendations, so that goals and prescriptions set by their care professionals will be achieved. In order to reach this objective, the METABO platform offers a Patient Feedback Module, which can be defined as the mobile-phone based graphical user interface that connects the user to the METABO functionalities. It includes input functions for measurement values, food intake, medication, and physical activity. It also comprises output functions, such as an electronic diabetic diary, automated warnings and reminders, prescriptions, and special functions to let the patient monitor his/her own progress. Finally, a function for direct messagecommunication with the medical doctor can be enabled on demand. Thus, the Patient Feedback Module covers, in a semi intelligent way, some educational, motivation-related, and objective needs of the diabetic patient.

There are existing mobile feedback solutions for diabetes patients, such as the DID by Rossi et al. [6], yet they are not platform based and thus isolated solutions. METABO is meant to be much more integrative.

There are some characteristics of Diabetes Mellitus that make it special among chronic diseases. These particular challenges have to be taken into account before applying the here presented system to any other chronic disease. First of all, the patients with type 1 and type 2 Diabetes have different needs regarding a patient support system. This is due to differences related to age and co-morbidities, motivation, and daily routines. Secondly, Diabetes Mellitus affects patients every day in core aspects of their lifestyle and the patients differ largely in their knowledge and their way to cope with it. When collecting user requirements in expert interviews and focus group discussions, we learned that even though they are often more proficient in topics such as nutrition, especially type 1 Diabetes Mellitus patients suffer from the restrictions that the disease imposes on their daily actions and thus from the attention that the disease requests from them on a daily basis. One patient told us: "Diabetes already rules $50 \%$ of my thoughts. I don't want it to be more" [7]. Furthermore, we understood that recently diagnosed patients, for example, struggle with learning about food and exercise related regulations, as well 
as with discovering the mechanisms that influence their personal glycaemic status, which are not the same in all patients. Thus, they will most often be more open to receiving advice and educational content than the experienced patients, who may intend to avoid Diabetesrelated topics whenever possible. Some patients are wellmotivated to follow their prescriptions, others are not. This motivation is not only different between patients: each patient goes through episodes of better or worse motivation, which a feedback system should properly respond to.

A proper management of Diabetes involves the control and precise reporting of the patient's food, physical exercise, measurements such as glycaemia, and medication, including insulin. Nowadays, patients use paper diaries to record their behavior and glycaemic status. METABO intends to replace the paper diary by a more intelligent electronic version, which is included in the feedback. Therefore, when giving feedback to patients, METABO needs to transport and visualize a lot of data. The complexity of the resulting architecture is due to the numerous actors involved and the number of intelligent feedback functions envisioned. Taking into account that data input functions, e.g. for food intake, are expected to be used several times every day, fast and uncomplicated dialogues are an important requirement. Proper filtering is a dire need in order to provide a usable system, as well as the installation of intelligent mechanisms to reduce the users' workload. Especially with respect to the patients' desire to spend as little time as possible on disease management, fast, direct, personalized and context adequate data input and feedback are of the essence. In addition to these visible aspects, it is important to manage the flow of these many pieces of information well.

We assume that the success of METABO will largely depend on its acceptance by the patients. Thus, the METABO Feedback system, which is the patient's primary user interface to the healthcare-related information and functions of METABO, needs to be highly useful and usable.

\section{METHODS}

In order to achieve the mentioned goal of empowering the patient, the telemedical application needs to improve the communication between patient and care-givers, and it should be customizable according to the patient's personal preferences, lifestyle, and knowledge deficits. It should empower the medical doctor to communicate more precisely and efficiently with the patient, instead of taking over too much of his/her responsibility.

Therefore, the METABO Feedback needs to meet several requirements:

1. The application needs to provide the most useful feedback contents possible. Thus, the feedback needs to prioritize the messages and send only information relevant in the specific situation and context. Furthermore, the users should be able to personalize the feedback by selecting the topics they want to be informed about.
2. The application should provide acceptable communication methods. So, education should be provided in a way that is fun to use. Furthermore, the application should provide various methods that enable the patient to monitor his/her progress - depending on his/her motivation and goals. This is, in the end, another personalization method.

3. METABO aims to support communication between Doctor and Patient instead of substituting it. The medical doctor should be enabled to follow and address the patient's behaviors directly. Thus, the feedback application takes advantage of the doctor's social influence.

4. Provide high usability. Taking into account that some users will need to use the METABO feedback every day, workload should be minimized in order to pay respect to their desire of spending the least possible time on health management. METABO should fit smoothly into their daily routines and require a minimum of learning.

Based on these challenges, the METABO Patient Feedback Module was defined.

As a consequence of the early user requirements definition process, the METABO patient group was divided into the two subgroups type 1 (T1) and type 2 (T2), represented by the fictive personas "Ana" (T1) and "George" (T2). This division resulted in the production of two distinct applications - one for each group. Ana's application was meant to be used by a pro-active user and thus designed to provide information on demand and to facilitate routine input of values. George's application should, on the contrary, be initiative, and motivate and support the user without him always asking for it. Both these feedback applications run on mobile devices, so that the patients can take them everywhere they go.

Another end device that we need to mention here is the medical doctor's PC, on which the "Control Panel" will run, thus including the medical doctor into the feedback loop. The Control Panel is important for the feedback process, as it serves for the medical doctor to define the patient's prescriptions, to enter the patient's data generated in the hospital context and to retrieve information about the patient's behavior and his/her health status, according to the data entered by the patient himself. Thus, the feedback that patients receive on their mobile device is partly generated by the application itself, taking into account the data entered by the patients; partly, it is based on automated decisions from the platform; and finally, one part is defined by the medical doctor.

Each of these two applications offers a range of different visualizations for the progress information of the patient. There are graphic tools to see, e.g., the weight loss over a certain period of time, or the relations between caloric intake and glycaemic measurement values. There are day and week views of the diary and a monthly overview on critical events, such as hypoglycemia.

Another example of METABO's user-intention-driven approach is the division of the health-education function into two concepts. For users who would go looking for a specific form of information in the moment they needed it, we came up with the ENCYCLOPEDIA. This is an easy-to-browse hypertext database. 
For users who would instead want to improve their general knowledge, either because of their own motivation or because this had been prescribed by the doctor, a serious game seemed to be much more attractive. Not only would learning be more fun, it would also provide the possibility to estimate the knowledge level of the user by the score and thus allow once more for a better adaptation of other feedback to this user. It has been shown that the usage of technology and media linked to gaming empowers attention, concentration and the memory of the users who are on the player role, and also offers a chance to the technology friendship for those people not used to technology, [8], [9]. The aim of encouraging the patient to enhance his/her knowledge about the disease and how to improve his/her health status through life style adaption was the driving force behind the integration of the METABO QUIZ.

Both parts of the educational content module, ENCYCLOPEDIA and QUIZ cover the contents Diet \& Nutrition, Hypoglycemia Episodes, Glycaemia, Insulin, Food Intake, Physical Activity, Blood Pressure, and Weight.

Another concept that spans over several diabetes topics is the GOAL Module [10]. In order to increase the patient's adherence to the prescriptions, the doctor can use the GOAL Module to set specific goals together with the patient, for example improving the knowledge about nutrition. The user can fulfill the goal by achieving a certain score in the QUIZ. If the user does not pursue his/her goal, METABO will remind the user of doing the QUIZ. The GOAL is meant to increase the specific motivation of the patient. Feedback messages reminding of the goal are expected to have a higher social importance, due to the contract with the physician.

Some users might just not be motivated to deal with certain diabetes topics. In order to ensure that METABO will not send too many unwanted messages, even if they seem appropriate from a treatment point-of-view, the users can manually select the topics that METABO will give support about. Users who know that they will, e.g., frequently fail to achieve their nutrition goals can customize METABO to send them only messages concerning glycaemia and physical activity.

The METABO Patient Feedback can be divided into two groups of components. The first group refers to the modules of the Feedback application that give the user access to data input functions, educational contents, or automated analyses of the contents in his/her electronic diary; these functions "triggered" or pulled by the user are called "PULL feedback". The PULL modules include, among others, the following: EDUCATION \& QUIZ Module, PRESCRIPTION Module and GOAL Module. Thus, PULL feedback is especially important for the pro-active user, who actively intents to put in or retrieve information when his/her current context requires it, for example when having lunch in an exotic restaurant.

The other group concerns the most innovatively managed feedback offered in METABO: The PUSH feedback. PUSH feedback comprehends all the feedback given by the initiative of the system. It works in three layers: L1, L2 and L3. Layer L1 comprehends the warnings. Warnings are urgent and health-critical messages, given for example when a patient has had lunch without confirming the intake of the necessary dose of insulin. Warnings must never fail to be delivered and thus be independent from connection problems. Warnings are therefore generated within the mobile application.

Then, there are the L2 and L3 PUSH contents: L2 messages are reminders that refer to patient behaviors which can only be assessed over a period of three to seven days. An example of an L2-message would be: "Did you measure your blood pressure during the last week? If yes, please insert the resulting values." L3 messages are related to the GOAL. An example for a patient who has agreed to lose a certain amount of weight in a specified time interval could be: "It is important to strictly follow your exercise prescription in order reach your goal!"

L2 and L3 feedback is handled by a so called Core Engine System (CES), an intelligent subsystem of the METABO platform. There are two reasons for that. First of all, it was realized that the METABO system would possibly send a lot of PUSH information to the user, due to its immense range of modules and data available. Thus, the system would also need to be intelligent enough to send not too many messages and to prioritize them well. The second reason is that patients who are, for example, very pro-active and compliant with their food prescriptions should not be receiving L2 and L3 messages concerning the topic of nutrition.

For these reasons, the CES was developed. The main task of the core engine is to harmonize the outputs generated as a consequence of medium- and long-term analyses of the patient's data input. In figure 1, the related communication process is depicted: The Core Engine receives the patient's data input (2) and returns reminders, goals-related messages and tips, without involving any other actor.

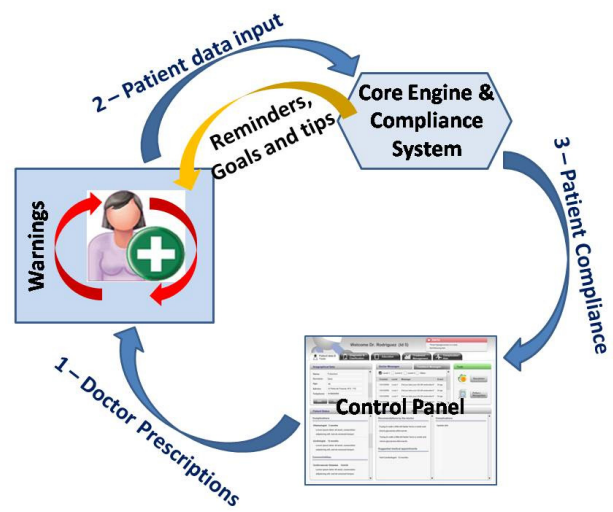

Fig. 1 The Patient Feedback Module in the loop

There is another feedback loop which can be seen in Figure 1: The COMPLIANCE system. It involves the patient, the METABO platform and the medical doctor. The system calculates two types of compliance: The system-usecompliance and the prescription-compliance. The first represents how often the patient makes the required use of the METABO system. For example: this compliance is low, 
if the user enters only one meal per day over a longer period of time. The latter represents the adherence to the prescriptions. If the patient frequently reports food intake that substantially exceeds his/her prescribed caloric values, this compliance value will be low. The patient's compliance values are only displayed in the medical doctor's control panel and are not used for the adaptations done by the Core Engine.

The Compliance system is therefore a good example of how METABO fosters communication between patient and doctor instead of replacing it: It is the doctor who checks the compliance values of the patient and accordingly tries to motivate the patient, adjusts his/her goals or changes prescriptions that seem to be too restrictive.

\section{RESULTS}

The above mentioned features have successfully been implemented in our prototypes, which are now to undergo a four week pilot test in three European hospitals. Within this section, we mention some typical Type 1 use cases that can be completed using these prototypes, and which are part of the pilot plan.

Via his control panel, the medical doctor can now enter food prescriptions for each patient, e.g. defining the recommended amount of carbohydrates to be consumed. The feedback application offers various ways of entering this information whenever the patient wants to do so. The design takes the different situations into account. The data entered by the patient is automatically analyzed by the central algorithms of METABO.

Type 1 patients will, for example, not receive any automated feedback messages if they enter their food correctly, and if furthermore the food quantities entered are in accordance to the prescription., However, if the patients passes days without entering such information, or if the patient keeps entering information that exceed the recommended daily carbohydrate target values, then the system will generate warnings and communicate with the patient through popup messages. The feedback strategy is different for Type 2 - patients: L3-messages, which refer to the GOAL, will always arrive, even if the patient is doing well.

\section{CONCLUSION}

Within this article, we have described the various strategies that serve for the adaptation of contents and communication strategies within the METABO Patient Feedback System:

1. Different visualization options for patient progress.

2. Encyclopedia and Quiz for patient education.

3. Warning generation in the local application.

4. Reminders, goals, and tips managed by the Core Engine System.

5. Message-topic setting options for the patient

6. Compliance algorithms to support the communication between patient and medical doctor.
The presented approach is in our opinion appropriate for the multiple actors and data sources integrated within the METABO platform, taking advantage of the different channels of interaction and thus meeting the most important user needs.

By the combined effort of the Compliance algorithms and the Core Engine, pro-active users are granted a minimum usage time of the METABO system while non-compliant users are supported in a personalized, context-adequate way. While the PUSH-function is adapted to the described circumstances, the PULL functions remain open for use to all users at any time, always allowing for pro-active behavior.

The technical implementation of these feedback strategies in the prototypes has been successful. If they truly meet the requirements of the users and if they lead to the expected amount of both compliance and satisfaction will only be known after the pilot phase.

\section{ACKNOWLEDGMENT}

The authors acknowledge the METABO consortium for their valuable contributions to this work. This project is partially funded by the European Commission (Contract Nr. 216270) under the $7^{\text {th }}$ Framework Program, Theme ICT2007.5.1, Personal Health Systems for Monitoring and Point-of-Care Diagnostics - Personalized Monitoring.

\section{REFERENCES}

[1] Alfred Winter, Reinhold Haux, Elske Ammenwerth, Birgit Brigl, Nils Hellrung and Franziska Jahn, "Health Information Systems". Health Informatics, 33-42, 2011.

[2] Bram L.T. Ramaekers, Josianne J. Janssen-Boyne, Anton P.M. Gorgels, Hubertus J.M. Vrijhoef, “Adherence Among Telemonitored Patients with Heart Failure to Pharmacological and Nonpharmacological Recommendations", Telemedicine and e-Health. July/August 2009.

[3] Frosch, D. L., Rincon, D., Ochoa, S. and Mangione, "Activating Seniors to Improve Chronic Disease Care: Results from a Pilot Intervention Study". Journal of the American Geriatrics Society, 58: 1496-1503. 2010,

[4] Paula M. Trief, Jeanne A. Teresi, Joseph P. Eimicke, Steven Shea, Ruth S. Weinstock, "Improvement in diabetes self-efficacy and glycaemic control using telemedicine in a sample of older, ethnically diverse individuals who have diabetes: the IDEATel project", Oxford Journals. 2008.

[5] European Commission. Information Society Technologies Program. METABO project. Chronic diseases related to metabolic disorders. ICT-26270. http://www.metabo-eu.org (Visited April 20, 2010).

[6] M. Rossi, A. Nicolucci, P. Di Bartolo, D. Bruttomesso, A. Girelli, F. Ampudia, D. Kerr, A. Ceriello, C. De La Questa Mayor, F. Pellegrini, D. Horwitz, G. Vespasiani, "Diabetes Interactive Diary: A New Telemedicine System Enabling Flexible Diet and Insulin Therapy While Improving Quality of Life", Diabetes Care, 2009.

[7] A. Guillén, D. Fernandez, G. Fico, I. Peinado, METABO Patient Centered Design. Internal Report, 2009.

[8] Marc Prensky, Digital game-based learning, 2001.

[9] Alberto Fuchslocher, Jörg Niesenhausa, Nicole Krämera, "Serious games for health: An empirical study of the game "Balance" for teenagers with diabetes mellitus", Entertainment Computing, 2010.

[10] G. Fico, A. Fioravanti, M.T. Arredondo, D. Ardigó, A. Guillén, "A Healthy Lifestyle Coaching-Persuasive Application for Patients with Type 2 Diabetes", 2010. 